\title{
An overview of penetrating traumatic brain injuries at a major civilian trauma centre in South Africa
}

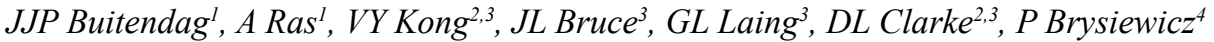 \\ ${ }^{1}$ Department of Surgery, Tygerberg Hospital, Stellenbosch University, Cape Town, South Africa \\ ${ }^{2}$ Department of Surgery, University of the Witwatersrand, Johannesburg, South Africa \\ ${ }^{3}$ Department of Surgery, University of KwaZulu Natal, Durban, South Africa \\ ${ }^{4}$ School of Public Health Medicine, University of KwaZulu Natal, Durban, South Africa
}

Corresponding author: Victor Kong (victorywkong@yahoo.com)

Background: This study reviews our experience with penetrating Traumatic Brain Injury (TBI) in order to define and describe the injury pattern and the outcome. A secondary aim of this study was to review the use of the Motor Score (M Score) and the Simplified Motor Score (SMS) to assess and triage patients with penetrating TBI.

Methods: All patients with a TBI secondary to a penetrating mechanism were identified from the Hybrid Electronic Medical Registry at Pietermaritzburg Metropolitan Trauma Service (PMTS) from January 2012 to December 2014. Standard demographic data, need for neuro-surgical intervention, location of external wounds, CT findings and mortality where analysed. The Glasgow Coma Scale (GCS) M score and SMS score were specifically evaluated to determine the relationship between the individual motor component and patient outcome.

Results: Over the two-year period January 2012-December 2014, a total of 384 patients were admitted following a penetrating TBI. There were 350 males and 34 females and of this total $7(1.82 \%)$ died. The mechanism of injury was axe (30), bottle (34), gunshot wound (GSW) (22) and stab wound (298). The average age for axe injuries was 27 and bottle injuries was 30 . The average age for firearms and knives was 29 and 30 respectively.

Surgery was not required for $76.67 \%$ of patients. The need for surgery varied according to mechanism of injury. Axe injuries were treated non-operatively in $47.83 \%$, bottle injuries in $87.50 \%$, firearms $70 \%$ and knife injuries were treated nonoperatively in $86.84 \%$ of cases.

The overall survival rate for a penetrating head injury in this population is $98.16 \%$. There were a total of 368 patients with a motor score of 6 of which one died. The survival rate was $99.7 \%$ and the mortality rate $0.3 \%$. There were only 6 patients with a motor score of 5 and only 2 with a motor score of 4 . The survival rate for both these groups was $100 \%$. There was a total of 6 patients with a motor score of 1 . There was a $100 \%$ mortality rate is this group.

Conclusion: Penetrating TBI has a good prognosis. The vast majority of cases do not require neuro-surgical intervention. Poor motor score is associated with a poor outcome.

S Afr J Surg 2019;57(1)

http://dx.doi.org/10.17159/2078-5151/2018/v57n1a2711

\section{Introduction}

Most traumatic brain injury (TBI) is secondary to blunt trauma and most of the literature on TBI has focused on this mechanism of injury. ${ }^{1,2}$ In addition, what literature there is on the topic has mostly emanated from the military and may not be directly relevant to the civilian setting. ${ }^{3-8}$ South Africa is a low to middle income country with significant discrepancies in wealth and access to health care and with a persistently high rate of trauma, with a major component of this being due to interpersonal violence..$^{2,7,8}$ This means that a significant proportion of TBI in South Africa is secondary to penetrating trauma, however this experience has not been documented in great detail. In light of this, we set out to review our experience with penetrating TBI in order to define and describe the injury pattern and the outcome of these patients in our setting. A secondary aim of this study was to review the use of the Motor Score (M Score) and the Simplified Motor Score (SMS) to assess and triage patients with penetrating TBI. 


\section{Setting}

All patients with penetrating TBI are managed by the staff of the Pietermaritzburg Metropolitan Trauma Service (PMTS). All patients with penetrating TBI are resuscitated according to Advanced Trauma Life Support (ATLS) principles. They receive supplemental oxygen and are intubated if they cannot maintain an airway. They are administered intravenous fluids and inotropes as required to maintain a mean arterial pressure of $65 \mathrm{mmhg}$ and they all receive prophylactic antibiotics as for a open fracture. Conservative management includes minor procedures such as suturing, washout and cleaning. All patients receive anti-epileptics as seizure prophylaxis for at least two weeks. Once the patient has been resuscitated and imaging scans have been obtained, these are discussed with staff at the neurosurgical service in Durban. During the neurosurgical consultation a decision is made with regard to the definitive management plan for the patient. Patients accepted by the neurosurgical service are either transported by road or airlifted to Durban, as appropriate.

The PMTS maintains a prospective digital trauma registry, which captures data at our institution. The admitting doctor clerks the patient onto an electronic proforma. This is the clerking process for all new trauma admissions - the clinical data is therefore entered in real-time. As the data is entered, it is directly incorporated into the registry. The completed pro forma is then printed out and becomes the patient's clinical record. Surgical Intervention, discharge and death results in updating of the registry. This system combines the functions of a medical registry and a medical record system. It also combines an electronic system with a paper-based system the hybrid electronic medical registry (HEMR).

\section{Methods}

All patients with a TBI as defined by injuries involving the cranium and breaching the dura mater secondary to a penetrating mechanism were identified from the HERM from January 2012 to December 2014. Standard demographic data were analysed as well as the following outcomes: need for neuro-surgical intervention, location of external wounds, CT findings and mortality.

The GCS M score was specifically evaluated to determine the relationship between the individual motor component and patient outcome. The M score was compared with risk of death and need for surgery. We also determined the sensitivity and specificity of the total GCS in predicting the following outcomes: need for intubation, presence of a significant finding on computed tomography (CT), need for surgery, and mortality. We went on to derive the SMS for each patient based on the recorded motor component of the GCS. An SMS of 0 was considered to be equivalent to a GCS M score of 1-4, an SMS of 1 to an M score of 5, and an SMS of 2 to an M score of 6. We compared the SMS with the overall GCS score and with the GCS M score for the same outcomes as listed above.

CT scan reports were reviewed and positive findings entered as part of patient records into the database. These were reorganized into 9 categories including Depressed Skull Fracture, Cerebral Contusion, Intra Cerebral Hemorrhage (ICH), Intraventricular Hemorrhage (IVH), Extradural Hematoma (EDH), SubDural Hematoma (SDH), Subarachnoid Hemorrhage (SAH), Free Air (Pneumocephalus) and the Weapon visible in situ.

Instruments used as weapons were classified into one of four broad categories including knife, axe, low velocity firearm and bottle.

\section{Statistical analysis}

Data were processed and analysed using Stata version 13.0 (StataCorp., USA). Differences in frequencies of categorical outcomes by GCS category were assessed using Pearson's $\chi 2$ test or Fisher's exact test if any expected cell count was $<5$ observations. Differences in mean GCS of classification or outcome (e.g. mortality) were assessed using Student's t-test. We constructed receiver operating characteristic (ROC) curves and measured the areas under these curves to compare the predictive value of the GCS, M score and SMS against, need for surgery, and mortality. We also calculated the $95 \%$ confidence intervals (CIs) of the areas under the curves for each outcome.

\section{Ethics}

Ethics approval to maintain the registry has been obtained from the Biomedical Research Ethics Committee (ref. no. BCA221/13 BREC) of the University of KwaZulu-Natal, Durban, and from the Research Unit of the Department of Health.

\section{Results}

Over the two-year period January 2012-December 2014, a total of 384 patients were admitted following a penetrating TBI. There were 350 males and 34 females and of this total $7(1.82 \%)$ died. The mechanism of injury was axe (30), bottle (34), low velocity GSW (22) and stab wound (298). The average age for axe injuries was 27 . The average age for bottle injuries was 29 . The average age for firearms and knives was 28 and 29 respectively.

\section{Location of external wounds}

Axe: $46.43 \%$ of these injuries involved the frontal area, $64.29 \%$ the parietal, $25.00 \%$ the occipital, and $10.71 \%$ the temporal area.

Bottle: $53.13 \%$ of these injuries involved the frontal area, $34.38 \%$ the parietal area, $15.63 \%$ the temporal area, and $6.25 \%$ the occipital area.

Low velocity firearm: $50 \%$ of injuries involved the frontal and parietal region, $10 \%$ the temporal region, and $5 \%$ the occipital region.

Knife: $50 \%$ of injuries involved the frontal region, $44.7 \%$ the parietal region, $21.05 \%$ the temporal region, and $5.26 \%$ the 


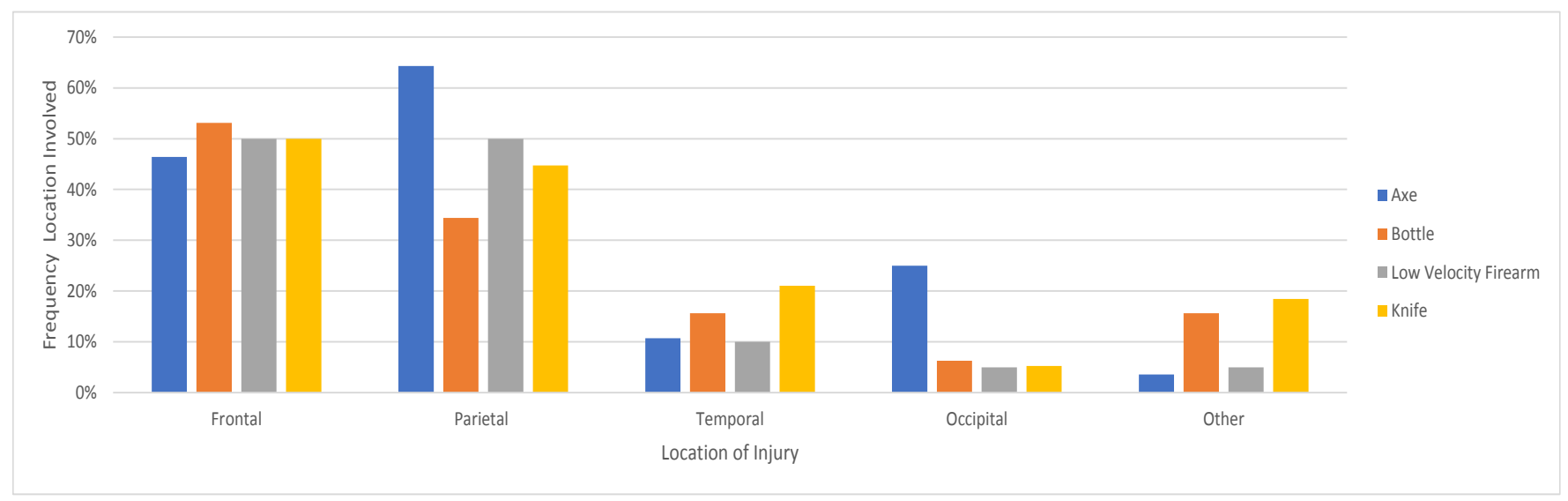

Figure 1. Location of Injury v. Weapon Used

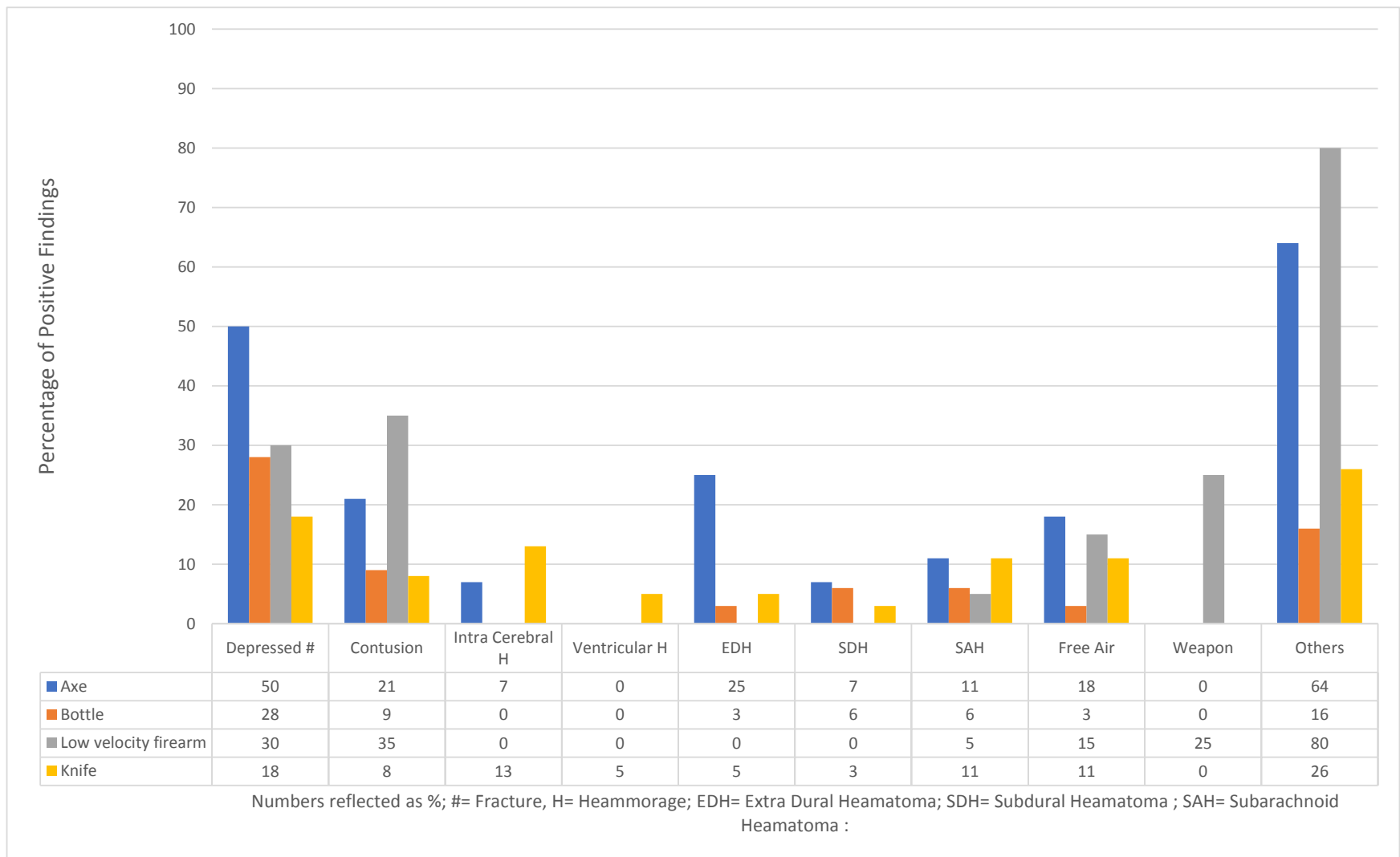

Figure 2. CT Findings v. Weapon Used

occipital region.

When the 4 weapon types were reviewed, the frontal and parietal regions were most frequently injured followed by the temporal region. Figure 1 and Table 1 show a graphical representation of this.

\section{CT Findings}

Axe: A total of $50 \%$ had a depressed skull fracture, $25 \%$ an extradural haematoma, $21.43 \%$ a contusion, $17.87 \%$ pneumocephalus, $10.7 \%$ a subarachnoid hemorrhage, $7.14 \%$ a subdural haematoma and $7.14 \%$ intra-cerebral hemorrhage
(ICH).

Bottle: A total of $28.13 \%$ had a depressed skull fracture, $9.38 \%$ a cerebral contusion, $6.25 \%$ a $\mathrm{SAH}, 6.25 \%$ a $\mathrm{SDH}$, $3.13 \%$ an EDH and $3.13 \%$ of cases had pneumocephalus.

Low velocity firearms: A total of $30 \%$ of these patients had depressed skull fractures, $45 \%$ had cerebral contusions, $15 \%$ had pneumocephalus, and $5 \%$ a SDH. In $25 \%$ of cases the bullet was in situ.

Knife: A total of $18.42 \%$ had depressed skull fractures $13.16 \%$ an ICH, $10.53 \%$ pneumocephalus, $10.53 \% \mathrm{SAH}, 7.89 \%$ a 


\begin{tabular}{lcccc} 
Table 1. Location of Injury v. Weapon Used & \\
\hline Location & Axe & Bottle & Low Velocity Firearm & Knife \\
\hline Frontal & $46,43 \%$ & $53,13 \%$ & $50,00 \%$ & $50,00 \%$ \\
Parietal & $64,29 \%$ & $34,38 \%$ & $50,00 \%$ & $44,74 \%$ \\
Temporal & $10,71 \%$ & $15,63 \%$ & $10,00 \%$ & $21,05 \%$ \\
Occipital & $25,00 \%$ & $6,25 \%$ & $5,00 \%$ & $5,26 \%$ \\
Other & $3,57 \%$ & $15,63 \%$ & $5,00 \%$ & $18,42 \%$
\end{tabular}

Table 2. Type of Surgery v. Weapon of Injury

\begin{tabular}{lcccc}
\hline Type of Surgery & Axe & Bottle & Knife & Firearm \\
\hline No surgery & $56,52 \%$ & $96,88 \%$ & $86,82 \%$ & $86,84 \%$ \\
Craniotomy & $26,09 \%$ & $3,13 \%$ & $6,20 \%$ & $7,89 \%$ \\
Debridement & $13,04 \%$ & $0,00 \%$ & $6,20 \%$ & $5,26 \%$ \\
Decompression & $4,35 \%$ & $0,00 \%$ & $0,78 \%$ & $0,00 \%$
\end{tabular}

contusion, 5.26\% a ventricular haemorrhage, 5.26\% an EDH, and $2.63 \%$ an SDH. There were no retained weapons in this cohort.

Figure 2 is a graphical representation of the findings. Picture 1 shows the CTB findings post assault with axe to head of one of the patients.

\section{Need for Neuro-Surgical Intervention}

Axe injuries were treated non-operatively in $56.52 \%$ of cases. $26.09 \%$ of them underwent craniotomies, $13.04 \%$ of axe injuries required debridement and only $4.35 \%$ of these cases underwent neuro-surgical decompression.

Bottle injuries were treated non-operatively in $96.88 \%$ of cases. This demonstrates the difference in force axe injuries and bottle injuries.

Low velocity firearms $86.84 \%$ of these cases were treated nonoperatively. Debridement and craniotomies were performed on these patients in $5 \%$ and $7.89 \%$ of cases respectively.

Knife injuries were treated non-operatively in $86.84 \%$ of cases. Craniotomies were performed on $6.2 \%$ of cases and debridement in $6.2 \%$ of cases. Decompression was done in $0.78 \%$ of the cases.

Table 2 tabulates and Figure 3 graphically represents the interventions required in this cohort of patients.

\section{Mortality}

Figure $4 \mathrm{~A}$ and $\mathrm{B}$ are linear graphs that show that the survival rate increases as the motor score (Figure 4A) and simplified motor score (Figure 4B) increases. The overall survival rate with penetrating head injuries in this population is $98.16 \%$. The mortality rate is therefore $1.83 \%$.

\section{Motor score as a predictor of mortality}

There was a total of 368 patients with a motor score of 6 of which one died. The survival rate was $99.7 \%$ and the mortality rate $0.3 \%$. There were only 6 patients with a motor score of 5 and only 2 with a motor score of 4 . The survival rate for both groups was $100 \%$. There was a total of 6 patients with a motor score of 1 . There was a $100 \%$ mortality rate is this group. See

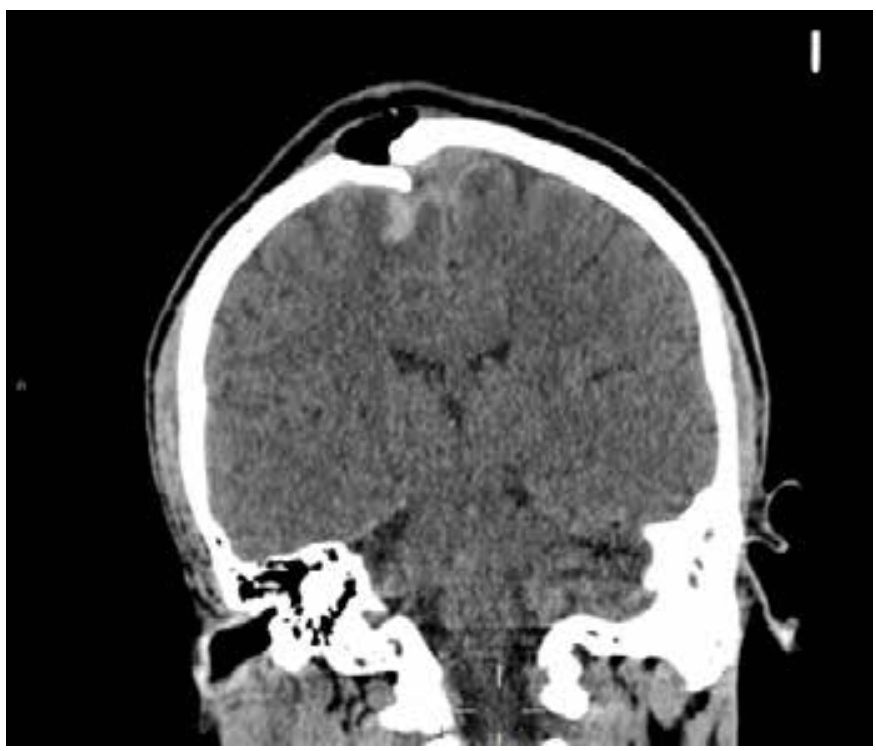

Picture 1. CT showing depressed skull fracture post assault with an axe

\section{Figure 4A.}

Figure 4B shows the simplified motor score versus the survival rate. The simplified motor score of 2 had a survival rate of $99.7 \%$. The mortality rate was $0.3 \%$. Simplified motor score of 1 had a total of 6 patients and all of them survived. The simplified motor score of 0 had 8 patients in total. The survival rate was only $25 \%$ and the mortality rate was $75 \%$.

\section{Discussion}

Cushing identified the principles of the surgical management of penetrating TBI which remained the gold standard during most of the major conflicts of the last century.3,9-11 These included removal of metallic and bone fragments, craniectomies to relieve ICP and debridement of non-viable cerebral tissue. This approach was challenged during the Israel Lebanon conflict in the early 1980s when military surgeons 


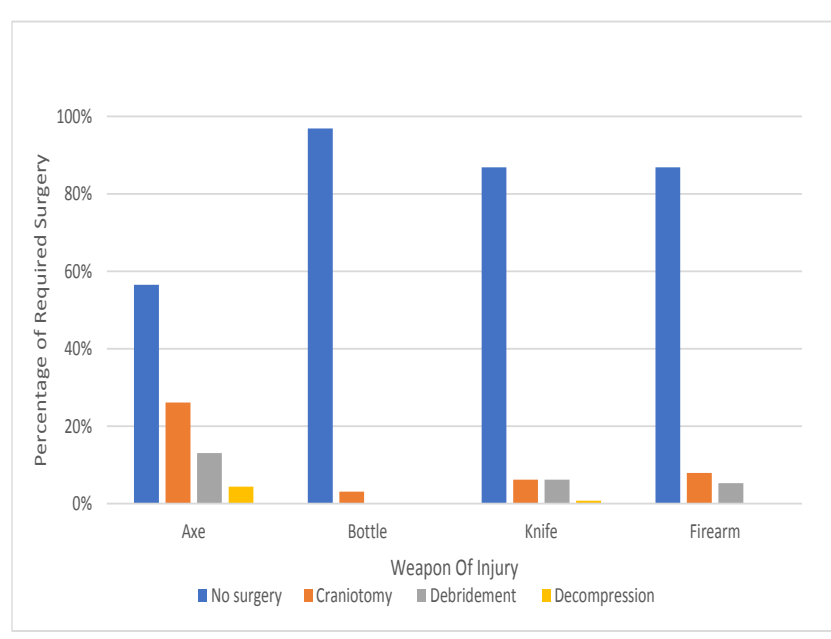

Figure 3. Surgery Type. Weapon used

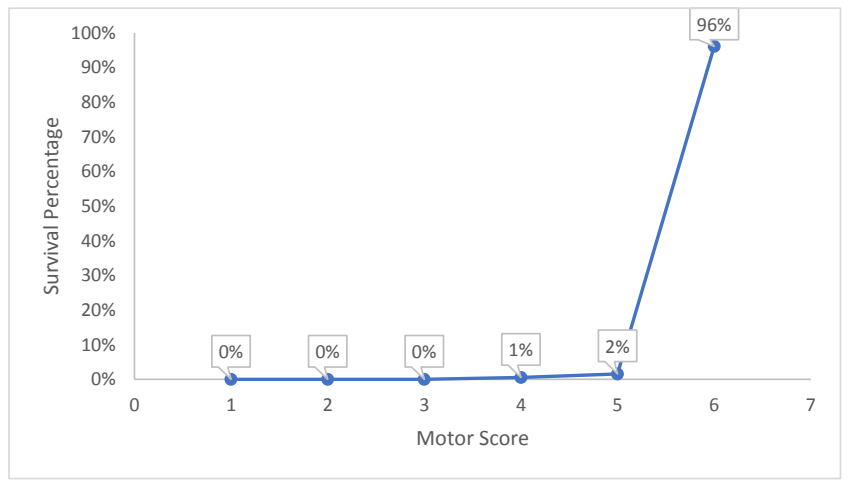

Figure 4a. Motor Score v. Survival

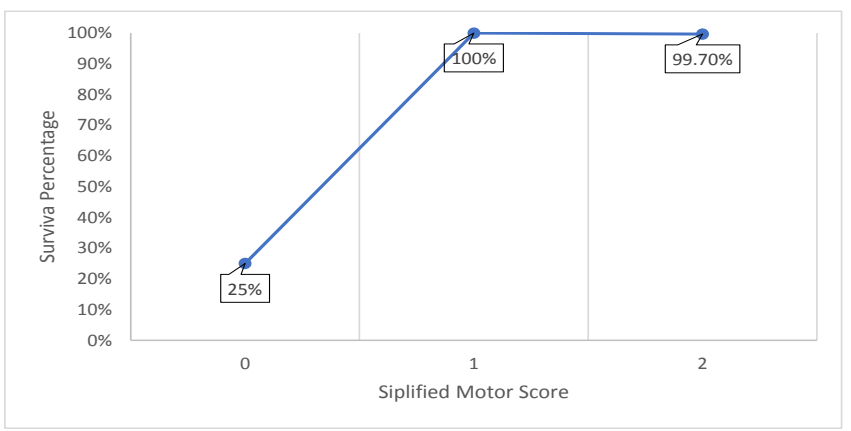

Figure 4b. Simplified Motor Score v. Survival

began to be more conservative in terms of debridement, in an effort to preserve cerebral tissue. The results of this more conservative approach were shown to be equivalent to earlier approaches and this conservative trend has persisted during more recent conflicts. ${ }^{4-6,12}$

Although our data is different to these military reports as the penetrating trauma is mostly of low velocity trauma, our results are very much in keeping with this trend towards conservatism. Only in patients with axe wounds to the head was surgery needed in more that $60 \%$ of cases. In all other forms of low velocity trauma, including GSW, the need for surgery was less that $30 \%$. Low velocity GSW were more likely to require surgery than stab and bottle injuries. Our low mortality rate is testament to the efficacy of this more conservative approach.

The use of the traditional GCS in the initial triage of patients with TBI has been criticised as it is complex and it is difficult to score the verbal and eye response in patents who have sustained facial and head trauma. ${ }^{13-15}$ We have recently demonstrated at our institution that the $M$ score and SMS can reliably predict a significant TBI as well as mortality in patients with blunt TBI. This study supports the use of motor score and the so-called Modified Motor Score in lieu of the formal GCS in patients with penetrating TBI.

\section{Study limitations}

This study has a number of limitations. These include the single centre nature of the study and the fact that some entries into the electronic database had missing data, and these patients could not be included.

\section{Conclusion}

We have documented the injury pattern associated with penetrating TBI in our setting. Most of these injuries do not require surgery and can be managed conservatively. The use of M score or Simplified Motor Score to identify patients with a poor prognosis seems to be appropriate.

\section{Acknowledgements}

None.

\section{Author contributions}

JJPB, AR, VYK, DLC designed the study, analysed the data and wrote the manuscript. JLB, GLL and PB reviewed the manuscript.

\section{Funding}

None.

\section{Conflicts of interest}

None. VYK, DLC, JLB GL are all current ATLS instructors.

\section{REFERENCES}

1. Meara J, Greenberg S. e Lancet Commission on Global Surgery Global surgery 2030: Evidence and solutions for achieving health, welfare and economic development. Surgery. 2015;157(5):834-5. Available from:https://doi.org/10.1016/j. surg.2015.02.009

2. Jerome E, Laing G, Bruce J, Sartorius B, Brysiewicz P, Clarke D. An audit of traumatic brain injury (TBI) in a busy developing-world trauma service exposes a significant deficit in resources available to manage severe TBI. S Afr Med J. 2017;107(7):621-5. Available at: https://doi.org/10.7196/ SAMJ.2017. v107i7.10562

3. Rosenfeld JV, Bell RS, Armonda R. Current concepts in penetrating and blast injury to the central nervous system. World J Surg. 2015;39:1352-62. Available at: https://doi.org/10.1007/ s00268-014-2874-7

4. Joseph B, Aziz H, Pandit V, Kulvatunyou N, O'Keeffe T, et al. 
Improving survival rates after civilian gunshot wounds to the brain. J Am Coll Surg. 2014;218:58-65. Available at: https://doi. org/10.1016/j.jamcollsurg.2013.08.018

5. Zafonte RD, Wood DL, Harrison-Felix CL, Millis SR, Valena NV. Severe penetrating head injury: a study of outcomes. Arch Phys Med Rehabil. 2001;82:306-10. Available at: https://doi. org/10.1053/apmr.2001.18226

6. Coronado VG, Xu L, Basavaraju SV, McGuire LC, Wald MM, et al. Surveillance for traumatic brain injury-related deathsUnited States, 1997-2007. MMWR Surveill Summ. 2011;60:132.

7. Laing G, Bruce J, Skinner D, Allorto N, Clarke D, Aldous C. Development, implementation, and evaluation of a hybrid electronic medical record system specifically designed for a developing world surgical service. World J Surg. 2013;38(6):1388-97. Available at: https://doi.org/10.1007/ s00268-013-2438-2

8. Laing G, Skinner D, Bruce J, Aldous C, Oosthuizen G, Clarke D. Understanding the burden and outcome of trauma care drives a new trauma systems model. World J Surg. 2014;38(7):16991706. Availabe at: https://doi.org/10.1007/s00268-014-2448-8

9. Jennett B, Teasdale G. Aspects of coma after severe head injury. Lancet. 1977;309(8017):878-81. Available at: https://doi.
org/10.1016/S0140-6736(77)91201-6

10. Teasdale G, Jennett B. Assessment of coma and impaired consciousness. Lancet. 1974;304(7872):81-4. Available at: https://doi.org/10.1016/S0140-6736(74)91639-0

11. Teasdale G, Jennett B. Assessment and prognosis of coma after head injury. Acta Neurochir. 1976;34(1-4):45-55. Available at: https://doi.org/10.1007/BF01405862

12. Aarabi B, Mossop C, Aarabi J. Surgical Management of civilian gunshot wounds to the head. Handb Clin Neurol. 2015;127:18193. Available at: https://doi.org/10.1016/B978-0-444-528926.00012-X

13. Choi S, Narayan R, Anderson R, Ward J. Enhanced specificity of prognosis in severe head injury. J Neurosurg. 1988;69(3):381-5. Available at: https://doi.org/10.3171/jns.1988.69.3.0381

14. Green S. Cheerio, laddie! Bidding farewell to the Glasgow Coma Scale. Ann Emerg Med. 2011;58(5):427-30. Available at: https://doi.org/10.1016/j.annemergmed.2011.06.009

15. Gill M, Windemuth R, Steele R, Green S. A comparison of the Glasgow Coma Scale score to simplified alternative scores for the prediction of traumatic brain injury outcomes. Ann Emerg Med. 2005;45(1):37-42. Available at: https://doi.org/10.1016/j. annemergmed.2004.07.429 